\title{
Development of Cerebral Blood Flow Volume in Preterm Neonates during the First Two Weeks of Life
}

\author{
MARTIN KEHRER, GUNNAR BLUMENSTOCK, STEFAN EHEHALT, RANGMAR GOELZ, \\ CHRISTIAN POETS, AND MARTIN SCHÖNING
}

Department of Child Neurology [M.K., S.E., M.S.], Department of Neonatology [R.G., C.P.], University Children's Hospital; and Department of Medical Information Processing [G.B.], University of Tübingen, D-72076 Tübingen, Germany

\begin{abstract}
To investigate the postnatal development of cerebral perfusion in preterm neonates with normal brains over the first $2 \mathrm{wk}$ of life, a prospective longitudinal study was designed. Quantitative measurement of cerebral blood flow (CBF) volume was performed using ultrasound flowmetry of the extracranial, brainfeeding arteries in 32 preterm infants of 28-35 wk gestational age. Measurements were done in the internal carotid and vertebral arteries of both sides on d 1, 2, 3, 7, and 14 after birth. A $10.0-\mathrm{MHz}$ linear transducer of a computed sonography system (Acuson 128/XP10) was used. Intravascular flow volumes were calculated as the product of angle-corrected time-averaged flow velocity and the cross-sectional area of the vessel. Mean CBF volume increased markedly over the first $2 \mathrm{wk}$. One-third of this rise already occurred from the first to the second postnatal day, thereafter there was a continuous increase from d 2 to $d 14$ of life. Whereas the absolute level of CBF volume was primarily determined by postmenstrual age, the pattern of postnatal changes in $\mathrm{CBF}$ volume was found to be independent of gestational age.
\end{abstract}

\section{ABSTRACT}

Arterial carbon dioxide tension, mean arterial blood pressure, and hematocrit had no influence on the development of CBF volume. The pronounced increase of CBF volume from $\mathrm{d} 1$ to $\mathrm{d}$ 2 is likely to represent a normal adaptive response of the cerebral circulation to postnatal life. The data presented here may serve as the basis for further studies to investigate whether deviations from this adaptive response are associated with an increased risk of brain injury. (Pediatr Res 58: 927-930, 2005)

Abbreviations
CBF, cerebral blood flow
ICA, internal carotid artery
MABP, mean arterial blood pressure
NIRS, near-infrared spectroscopy
Paco $_{2}$, arterial carbon dioxide tension
TAV, time-averaged velocity
VA, vertebral artery

However, quantitative Doppler measurement of CBF volume is not possible with this method, because it is impossible to determine the diameter of the intracranial vessels.

Until now, quantitative CBF measurement in preterm neonates has been limited to the ${ }^{133}$ xenon-clearance technique (7), $\mathrm{H}_{2}{ }^{15} \mathrm{O}$-positron-emission-tomography $(8,9)$, or NIRS (10). These methods are technically difficult and generally not feasible for clinical use. The ${ }^{133}$ xenon-clearance technique and PET are not widely available and involve ionizing radiation, whereas quantitative NIRS measurements are limited to a subgroup of mechanically ventilated neonates for methodological reasons. As a consequence, quantitative data on the postnatal development of CBF are scant (11-13).

Recently, we were able to show that quantitative $\mathrm{CBF}$ volume can be measured (in milliliters per minute) by color duplex sonography of the extracranial cerebral arteries in neonates (14). Estimated CBF values obtained with this method (15) correspond to neonatal CBF values determined
Received January 13, 2005; accepted April 18, 2005.

Correspondence: Martin Schöning, M.D., Department of Child Neurology, University Children's Hospital, Hoppe-Seyler-Straße 1, D-72076 Tübingen, Germany; e-mail: martin.schoening@med.uni-tuebingen.de

This study was supported by the University of Tübingen (Grant fortüne 1076-0-0).

DOI: 10.1203/01.PDR.0000182579.52820.C3 
with other, more invasive techniques $(7,8,10)$. A crosssectional study in 113 preterm and term neonates revealed a marked rise in CBF volume with increasing postmenstrual age (15).

The aim of the present longitudinal study was to investigate the postnatal development of cerebral perfusion in preterm neonates with normal brains by serial measurements during the first $14 \mathrm{~d}$ of life.

\section{METHODS}

Study group. In a prospective study, 35 preterm newborns admitted to the neonatal intensive care unit of the University Children's Hospital Tübingen were examined. Three of them were excluded from the present analysis because of clinical details that could have a relevant influence on cerebral perfusion (severe asphyxia after placental abruption, infarction of the middle cerebral artery, and application of indomethacin, respectively). The final study group consisted of 32 preterm newborns (17 male) born at 28.4-35.0 (median, 33.0) wk of gestational age. The ranges (and medians) of birth weight and head circumference were 940-2660 (1835) $\mathrm{g}$ and 25.0-33.0 (30.0) $\mathrm{cm}$. Cerebral B-mode ultrasound examinations were normal in all cases during the follow-up study and at the time of discharge. None of the neonates had ever presented neurologic abnormalities.

With the exception of one child that was mechanically ventilated during the first day of life after administration of surfactant, all neonates were breathing spontaneously at all the examinations. Eighteen infants were temporarily treated with continuous positive airway pressure and/or supplementary oxygen. None of the infants was receiving medication that was likely to affect cerebral circulation. Informed consent was given by the parents in all cases. The study was approved by the Ethics Committee of the University of Tübingen.

MABP was measured noninvasively by an oscillometric technique using an inflatable cuff (Philips Intellivue MP70 Neonatal, Philips Medical Systems, Andover, MA). Estimates of $\mathrm{PaCO}_{2}$ were obtained using a transcutaneous monitor (Linde Micro Gas 7650 rapid, Linde Medical Sensors AG, Basel, Switzerland) calibrated with blood gas samples. MABP, $\mathrm{PaCO}_{2}$, and hematocrit were monitored as clinically required.

Study protocol. Color duplex sonography of all the extracranial cerebral arteries, i.e. the ICA and VA of both sides, was performed over the first $2 \mathrm{wk}$ of life according to the following schedule: A total of five single measurements of CBF volume were performed, one on each of the first $3 \mathrm{~d}$ of life, one on $\mathrm{d}$ 7 , and one on $\mathrm{d}$ 14. In all cases, the examinations on the first day of life were performed after the postnatal age of $5 \mathrm{~h}$. On the first $3 \mathrm{~d}$ of life, we attempted to keep the intervals between the examinations as close to $24 \mathrm{~h}$ as possible. All measurements were performed by the same examiner (M.K.).

Examination procedure. In all infants, measurements were performed at the bedside in the neonatal intensive care unit while the children slept. The examination of all the extracranial arteries required 7-36 (median, 14) min The subjects lay in a supine position with their heads slightly elevated and turned to the contralateral side by $\sim 25^{\circ}$.

Measurements were made with a 10.0-MHz linear transducer (aperture size $26 \mathrm{~mm}$ ) of a computed sonography system (Acuson 128/XP10, Mountain View, California) at a location at least $1.0 \mathrm{~cm}$ above the bifurcation in the ICA. The VA was examined in the transverse part (V2-segment), generally between the levels $\mathrm{C} 3$ and $\mathrm{C} 5$.

The luminal diameter of the vessels, d, was measured on a magnified, high-resolution B-mode image between the bright internal layers of the parallel vessel walls at the exact site of color duplex measurement and perpendicular to the artery's longitudinal axis. The calipers could be adjusted in $0.1-\mathrm{mm}$ increments. The mean of duplicate luminal measurements was calculated for each vessel. The color duplex sonography procedure consisted of acquiring a color Doppler image of the vessel and carrying out an angle-corrected pulsed Doppler time-frequency analysis of the intravascular flow in each artery. At a site of undisturbed color Doppler flow, the pulsed Doppler sample volume was positioned over the entire width of the vessel. The electronic steering feature of the linear probe was used to keep the angle of insonation as low as possible, in most cases between $20^{\circ}$ and $45^{\circ}$ in the ICA, and between $40^{\circ}$ and $55^{\circ}$ in the VA. Visual checks on the maximal luminal width and acoustic checks on the optimum time frequency Doppler signal served to ensure that the sample volume passed through the center of the vessel. A spectral curve was then recorded with the color Doppler image "frozen." The Doppler signal was evaluated only if it was stable for at least $5 \mathrm{~s}$ and did not contain bidirectional frequencies (which would indicate disturbed flow). The angle of insonation was adjusted in $1^{\circ}$ increments by aligning an angle indicator parallel to the walls of the vessels and along the intravascular color Doppler stream.
Angle-corrected time-averaged flow velocity (TAV), i.e. the integral of the mean flow velocities of all moving particles passing the sample volume, was measured over three to five complete cardiac cycles. In this way, the pulsatile parabolic flow is mathematically transformed into a continuous plug flow. The intravascular flow volume FV can be determined by calculating the product of the angle-corrected TAV and the cross-sectional area of the circular vessel A with the formula $\mathrm{FV}=\mathrm{TAV} * \mathrm{~A}=\mathrm{TAV} *\left[(\mathrm{~d} / 2)^{2} * \pi\right] . \mathrm{CBF}$ volume was determined by adding the bilateral ICA and VA flow volumes, i.e. the integral of flow passing through the anterior and posterior brain circulation.

Statistics. Statistical analysis was performed with the program "JMP" (version 4.0.5, SAS Institute, Cary, NC). Postnatal changes in CBF volume were analyzed by repeated-measures ANOVA with time as an explanatory variable. Two-factorial analysis was used to compare the patterns of change for different gestational-age groups. Additionally, day-to-day-differences between study time points were calculated and tested. Furthermore, the dependence of $\mathrm{CBF}$ volume on postnatal age, gestational age, $\mathrm{PaCO}_{2}, \mathrm{MABP}$, and hematocrit was analyzed using stepwise multiple linear regression analysis. Significance was set at $p<0.05$.

\section{RESULTS}

A complete set of five examinations could be performed in 29 out of the 32 infants. Two infants were transferred to another hospital after $\mathrm{d} 3$, and one was discharged after $\mathrm{d} 7$. In total, $155 \mathrm{CBF}$ volume measurements could be analyzed. As shown in Figure 1, mean CBF volume increased over the first $14 \mathrm{~d}$ of life (repeated-measures ANOVA: time effect, $p=$ 0.0002). In particular, there was a marked increase in $\mathrm{CBF}$ volume from the first to the second day of life (Figure 1, Table 1). Infants born between 28 and 31 wk had lower $\mathrm{CBF}$ volumes than those born between 32 and $35 \mathrm{wk}$ of gestational age at all study time points (Figure 2; ANOVA: gestational-age group effect, $p<0.0001)$. However, the dynamics of CBF volume increase was identical for infants in both gestational-age groups (ANOVA: interactions, NS). Stepwise multiple linear regression analysis revealed that the increase in CBF volume depended on postnatal age $(p=0.0095)$ and gestational age $(p<0.0001)$, but not on variations of $\mathrm{PaCO}_{2}, \mathrm{MABP}$, or hematocrit.

\section{DISCUSSION}

Cerebral perfusion rises markedly during the first $2 \mathrm{wk}$ of life in preterm neonates. The most pronounced increase can be observed from the first to the second day of life. Thereafter, a continuous rise occurs from d 2 to $\mathrm{d} 14$. By combining the

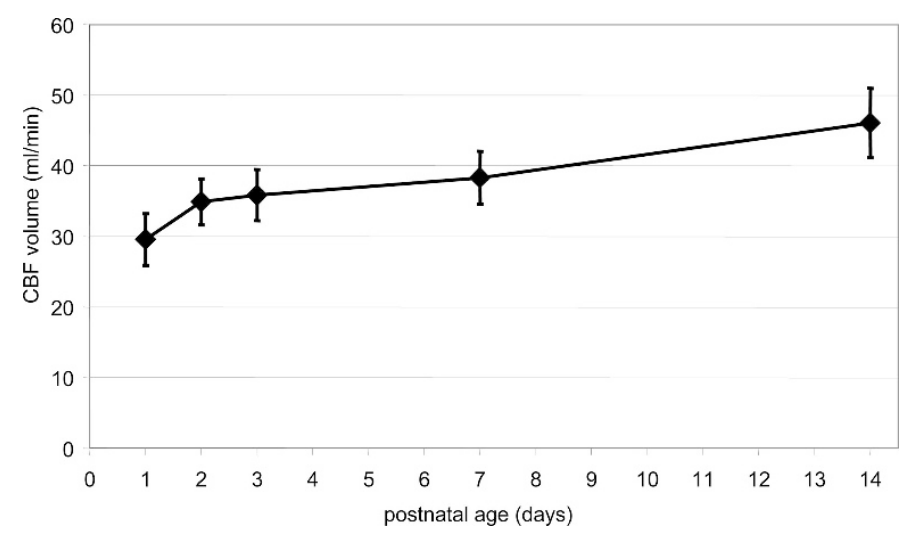

Figure 1. Development of CBF volume with increasing postnatal age. Mean and $95 \%$ confidence interval are shown (repeated-measures ANOVA; $n=29$, $p=0.0002)$. 
Table 1. Day-to-day-differences between the time points of the study

\begin{tabular}{|c|c|c|c|}
\hline $\begin{array}{c}\text { Compared } \\
\text { days }\end{array}$ & $\begin{array}{l}\text { Difference in mean } \\
\text { CBF volume } \\
(\mathrm{mL} / \mathrm{min} \pm \mathrm{SD})\end{array}$ & $p$ Value* & $\begin{array}{c}\text { Average increase } \\
\text { in } \mathrm{CBF} \text { volume } \\
\text { per day } \\
(\mathrm{mL} / \mathrm{min})\end{array}$ \\
\hline $\mathrm{d} 2-\mathrm{d} 1$ & $5.3 \pm 7.2$ & $<0.001$ & 5.3 \\
\hline $\mathrm{d} 3-\mathrm{d} 2$ & $0.9 \pm 7.3$ & NS (0.48) & 0.9 \\
\hline $\mathrm{d} 7-\mathrm{d} 3$ & $2.1 \pm 10.4$ & NS (0.28) & 0.5 \\
\hline $\mathrm{d} 14-\mathrm{d} 7$ & $7.1 \pm 12.7$ & 0.006 & 1.0 \\
\hline
\end{tabular}

* Post hoc day-to-day comparisons based on the paired $t$ test.

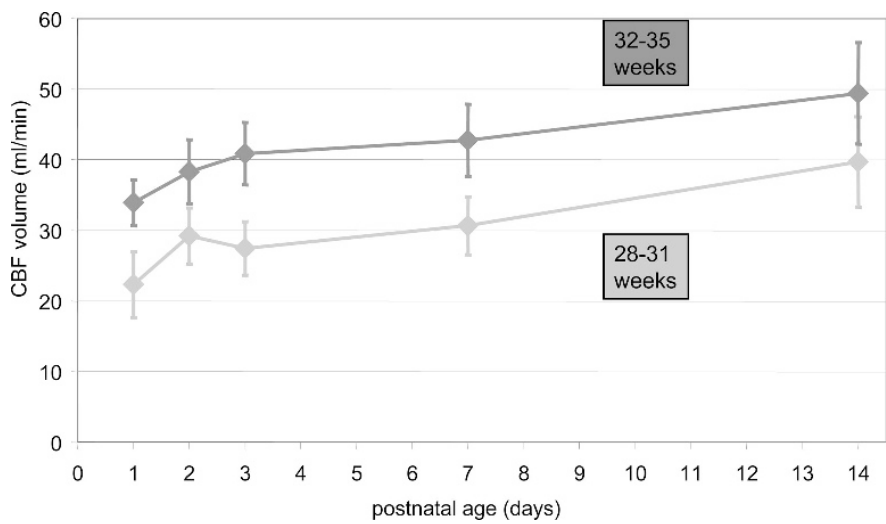

Figure 2. Development of CBF volume with increasing postnatal age in two different gestational age groups. Mean and $95 \%$ confidence interval are shown (ANOVA; $n=29, p<0.0001$ ).

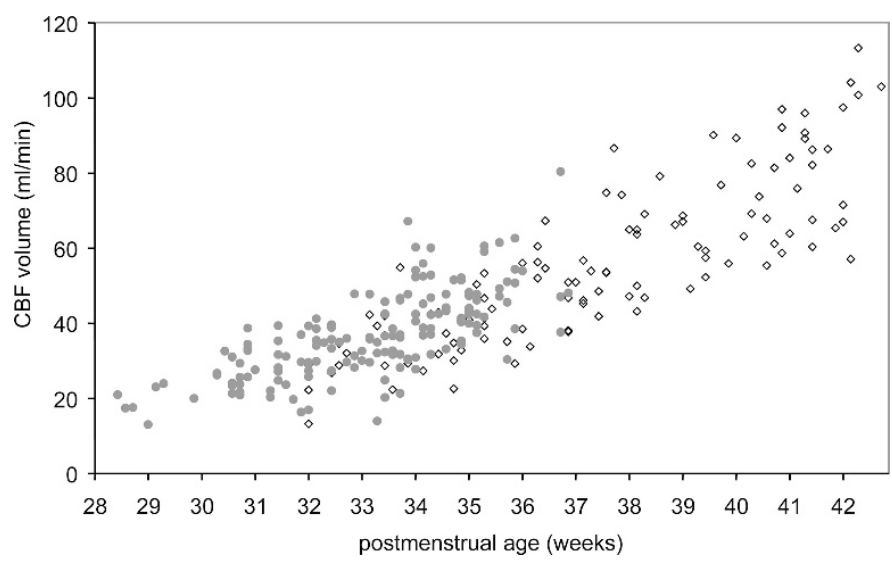

Figure 3. Development of CBF volume with increasing postmenstrual age. Open dots: Reference data on CBF volume $(n=113)$ [cross-sectional study (15)]. Closed dots: CBF volume data from the present study $(n=155)$.

results of this longitudinal study with those of a previous cross-sectional study on 113 preterm and term neonates (15), the impact of postnatal versus that of postmenstrual age on CBF volume can now be analyzed in detail (Figure 3). The present CBF volume data obtained from the 2nd to the 14th postnatal day fit the postmenstrual-age-specific reference data established in the cross-sectional study exactly. In addition, the dynamics of $\mathrm{CBF}$ volume increase over the course of the first 2 wk was identical in both studies. This continuous rise in CBF volume with increasing postmenstrual age has been shown to be due to increases in both brain weight and perfusion (15).
In contrast, the steep rise in $\mathrm{CBF}$ volume from the first to the second day of life observed in this study is exclusively due to an increase in cerebral perfusion, as there is no increase in brain weight during this short time period. Furthermore, we found that the early rise in CBF volume from the first to the second day of life was independent of gestational age.

Many studies have shown a postnatal increase in intracranial blood flow velocities in preterm and term neonates $(3,13,16-$ 19). However, the basic limitation of Doppler sonography of intracranial vessels is that it provides only a measure of flow velocity and not of flow volume because it is impossible to measure the diameter of the insonated blood vessel.

The postnatal increase in $\mathrm{CBF}$ volume in preterm neonates is consistent with CBF measurements acquired using the ${ }^{133} \mathrm{xe}-$ non-clearance technique (11) or NIRS $(12,13)$. But these data can hardly be compared with ours, as i) all infants included in these studies were on artificial ventilation, ii) some were paralyzed with pancuronium and sedated with phenobarbital (11), iii) some neonates suffered from hemorrhagic or ischemic lesions (13), and iv) observations were confined to the first 2 $(13)$ or $3(11,12) \mathrm{d}$ of life.

In the present study, the postnatal increase in CBF volume was not influenced by variations of $\mathrm{MABP}, \mathrm{PaCO}_{2}$, and hematocrit. Likewise, quantitative NIRS measurements of CBF over the first $3 \mathrm{~d}$ of life on 11 extremely preterm, mechanically ventilated neonates revealed a significant rise in $\mathrm{CBF}$ that was independent of $\mathrm{MABP}, \mathrm{PaCO}_{2}$, or hematocrit (12). On the other hand, CBF measurements using the ${ }^{133}$ xenon-clearance technique suggested that the postnatal increase in $\mathrm{CBF}$ is correlated with changes in $\mathrm{PaCO}_{2}$ and $\mathrm{Hb}$ concentration (11). Our results corroborate the findings of Meek et al. (12), and we agree with these authors that the increase in CBF volume from the first to the second day of life is likely to represent a normal adaptive response of the cerebral circulation to postnatal life.

In this prospective follow-up study, ultrasound flowmetry of the extracranial brain-feeding arteries proved to meet all the criteria for a useful tool for serial measurements and monitoring of the cerebral circulation in preterm infants: All the measurements (on each child and each day) could be completed successfully. This technique is noninvasive and innocuous; it can be performed at the bedside in the neonatal intensive care unit and repeated as often as desired. One limitation of this method is that the time required to complete a study (on the average 10-15 $\mathrm{min}$ ) makes it unsuited for measuring rapid changes or fluctuations in cerebral perfusion. Furthermore, measurements are not possible in restless infants and hence have to be performed on neonates while they are asleep. As this technique is operator-dependent, meticulous measurement technique must be observed. Ultimately, however, we were able to show that $\mathrm{CBF}$ volume measurements in neonates were highly reproducible by two independent observers (20).

This first study with serial, quantitative CBF volume measurements in preterm infants enables us to delineate the postnatal development of cerebral perfusion over the first $14 \mathrm{~d}$ of life. The most pronounced increase in $\mathrm{CBF}$, occurring between the first and the second day of life in infants with normal brains, is likely to represent a normal adaptive response of the 
cerebral circulation to postnatal conditions. It coincides with the very time period of the greatest risk of intraventricular hemorrhage in preterm neonates. Whereas the absolute initial CBF volume is primarily determined by gestational age, the developmental pattern of CBF volume after birth seems to be independent of gestational age. The data presented here may serve as the basis for further studies to investigate whether deviations from this adaptive response are associated with an increased risk of brain injury.

\section{REFERENCES}

1. Krägeloh-Mann I, Toft P, Lunding J, Andresen J, Pryds O, Lou HC 1999 Brain lesions in preterms: origin, consequences and compensation. Acta Paediatr 88:897908

2. Volpe JJ 2001 Neurobiology of the periventricular leukomalacia in the premature infant. Pediatr Res 50:553-562

3. Volpe JJ 2001 Neurology of the Newborn. WB Saunders, Philadelphia, pp 156-493

4. Tsuji M, Saul JP, du Plessis A, Eichenwald E, Sobh J, Crocker R, Volpe JJ 2000 Cerebral intravascular oxygenation correlates with mean arterial pressure in critically ill premature infants. Pediatrics 106:625-632

5. Hagberg H, Peebles D, Mallard C 2002 Models of white matter injury: comparison of infectious, hypoxic-ischemic, and excitotoxic insults. Ment Retard Dev Disabil Res Rev 8:30-38

6. Meek JH, Tyszczuk L, Elwell CE, Wyatt JS 1999 Low cerebral blood flow is a risk factor for severe intraventricular haemorrhage. Arch Dis Child Fetal Neonatal Ed 81:F15-F18

7. Greisen G 1986 Cerebral blood flow in preterm infants during the first week of life. Acta Paediatr Scand 75:43-51
8. Altman DI, Powers WJ, Perlman JM, Herscovitch P, Volpe SL, Volpe JJ 1988 Cerebral blood flow requirement for brain viability in newborn infants is lower than in adults. Ann Neurol 24:218-226

9. Altman DI, Perlman JM, Volpe JJ, Powers WJ 1993 Cerebral oxygen metabolism in newborns. Pediatrics 92:99-104

10. Edwards AD, Wyatt JS, Richardson C, Delpy DT, Cope M, Reynolds EO 1988 Cotside measurement of cerebral blood flow in ill newborn infants by near infrared spectroscopy. Lancet 2:770-771

11. Pryds O, Greisen G 1989 Effect of PaCO2 and haemoglobin concentration on day to day variation of $\mathrm{CBF}$ in preterm neonates. Acta Paediatr Scand Suppl 360:33-36

12. Meek JH, Tyszczuk L, Elwell CE, Wyatt JS 1998 Cerebral blood flow increases over the first three days of life in extremely preterm neonates. Arch Dis Child Fetal Neonatal Ed 78:F33-F37

13. Pellicer A, Valverde E, Gayá F, Quero J, Cabañas F 2001 Postnatal adaptation of brain circulation in preterm infants. Pediatr Neurol 24:103-109

14. Kehrer M, Goelz R, Krägeloh-Mann I, Schöning M 2002 Measurement of volume of cerebral blood flow in healthy preterm and term neonates with ultrasound. Lancet 360:1749-1750

15. Kehrer M, Krägeloh-Mann I, Goelz R, Schöning M 2003 The development of cerebral perfusion in healthy preterm and term neonates. Neuropediatrics 34:281-286

16. Bode H, Wais U 1988 Age dependence of flow velocities in basal cerebral arteries. Arch Dis Child 63:606-611

17. Winberg P, Sonesson SE, Lundell BP 1990 Postnatal changes in intracranial blood flow velocity in preterm infants. Acta Paediatr Scand 79:1150-1155

18. Yanowitz TD, Yao AC, Pettigrew KD, Werner JC, Oh W, Stonestreet BS 1999 Postnatal hemodynamic changes in very-low-birthweight infants. J Appl Physiol 87:370-380

19. Yoshida H, Yasuhara A, Kobayashi Y 1991 Transcranial doppler sonographic studies of cerebral blood flow velocity in neonates. Pediatr Neurol 7:105-110

20. Ehehalt S, Kehrer M, Goelz R, Poets C, Schöning M 2005 Cerebral blood flow volume measurements with ultrasound: interobserver reproducibility in preterm and term neonates. Ultrasound Med Biol 31:191-196 